\title{
Formulation of phase-field energies for microstructure in complex crystal structures
}

\author{
Lun Yang (杨伦) ${ }^{\mathrm{a})}$ and Kaushik Dayal ${ }^{\mathrm{b})}$ \\ Mechanics, Materials, and Computing, Civil and Environmental Engineering, Carnegie Mellon University, \\ Pittsburgh, Pennsylvania 15213, USA
}

(Received 25 November 2009; accepted 19 January 2010; published online 26 February 2010)

\begin{abstract}
The unusual properties of many multifunctional materials originate from a structural phase transformation and consequent martensitic microstructure. Phase-field models are typically used to predict the formation of microstructural patterns and subsequent evolution under applied loads. However, formulating a phase-field energy with the correct equilibrium crystal structures and that also respects the crystallographic symmetry is a formidable task in complex materials. This paper presents a simple method to construct such energy density functions for phase-field modeling. The method can handle complex equilibrium structures and crystallographic symmetry with ease. We demonstrate it on a shape memory alloy with 12 monoclinic variants. () 2010 American Institute of Physics. [doi:10.1063/1.3319503]
\end{abstract}

The unusual behavior of shape-memory alloys, ferroelectrics, and other multifunctional materials is driven by a structural phase transformation. Below the critical phasetransformation temperature, the crystal structure changes and this leads to multiple, symmetry-related variants. The different variants can form microstructural mixtures to satisfy applied boundary conditions. Changes in the applied loads can cause microstructural rearrangements rather than the lattice distortions that are characteristic of typical materials. This ability to rearrange microstructure leads to unusual and technologically important behavior as seen in shape-memory alloys, ferroelectrics, and other multifunctional materials. ${ }^{1-4}$

To enable design with multifunctional materials, it is important to be able to predict the microstructural patterns. Phase field models are widely used for this purpose., ${ }^{5,11-13}$ They obtain microstructural patterns by minimizing the free energy $E$ of a specimen $\Omega$. In the case of shape-memory alloys, that are the focus of this paper, $E$ consists of $W_{\text {inter }}$, the surface energy of the interfaces between different variants, and $W_{\text {aniso }}$, the anisotropy energy that penalizes deviation of the strain $\boldsymbol{\epsilon}(\mathbf{x})$ from the crystallographically preferred states.

$$
E[\boldsymbol{\epsilon}]=\int_{\Omega}\left[W_{\text {inter }}(\nabla \boldsymbol{\epsilon})+W_{\text {aniso }}(\boldsymbol{\epsilon})\right] d \mathbf{x} .
$$

$W_{\text {inter }}=A_{0}|\nabla \boldsymbol{\epsilon}|^{2} / 2$ penalizes the gradient of the strain and hence the interface has finite thickness to allow easy computation. In general, constructing $W_{\text {aniso }}$ can be challenging; ${ }^{7,14,15}$ it must have the appropriate minima in a high-dimensional space as well as reflect the symmetry of the crystal. This letter shows a simple method to construct $W_{\text {aniso }}$ by introducing a set of auxiliary scalar fields $\lambda_{I}(\mathbf{x})$. We also demonstrate the approach by calculating the microstructure in thin-film shape memory alloy NiTi with monoclinic symmetry that is of technological importance to small-scale actuators. ${ }^{16}$

We start by introducing the scalar fields $\lambda_{I}(\mathbf{x}), I$ $=1, \ldots, N$ where there are $N$ variants. Each variant is associ-

\footnotetext{
a) Electronic mail: luny@andrew.cmu.edu.

b)Electronic mail: kaushik@cmu.edu.
}

ated with a tensor $\boldsymbol{\epsilon}_{0}^{I}$ that represents the stress-free strain. At every point, we define the average stress-free strain to be $\boldsymbol{\epsilon}_{0}(\mathbf{x})=\sum_{I=1}^{N} \lambda_{I}(\mathbf{x}) \boldsymbol{\epsilon}_{0}^{I}$.

We write the free energy of a shape-memory specimen in terms of $\boldsymbol{\epsilon}(\mathbf{x})$ and the set $\left\{\lambda_{I}(\mathbf{x})\right\}$ as follows:

$$
\begin{aligned}
& E\left[\boldsymbol{\epsilon}(\mathbf{x}),\left\{\lambda_{I}(\mathbf{x})\right\}\right] \\
& \quad=\int_{\Omega}\left[W_{\text {inter }}\left(\boldsymbol{\epsilon},\left\{\lambda_{I}(\mathbf{x})\right\}\right)+W_{\text {aniso }}\left(\boldsymbol{\epsilon},\left\{\lambda_{I}(\mathbf{x})\right\}\right)\right] d \mathbf{x} .
\end{aligned}
$$

For the interfacial energy density we use $W_{\text {inter }}$ $=A_{0} \Sigma_{I=1}^{N}\left|\nabla \lambda_{I}(\mathbf{x})\right|^{2} / 2$. We write $W_{\text {aniso }}$ as the sum of following three terms:

$$
\begin{aligned}
& \underbrace{\frac{1}{2}\left[\boldsymbol{\epsilon}-\boldsymbol{\epsilon}_{0}(\mathbf{x})\right]: \mathbf{C}:\left[\boldsymbol{\epsilon}-\boldsymbol{\epsilon}_{0}(\mathbf{x})\right]}_{\text {Linear elastic penalty for } \boldsymbol{\epsilon} \neq \boldsymbol{\epsilon}_{0}(\mathbf{x})}+\underbrace{A \sum_{I=1}^{N} \lambda_{I}(\mathbf{x})^{2}\left[1-\lambda_{I}(\mathbf{x})\right]^{2}}_{\text {Drives each } \lambda_{I} \text { to lor } 0} \\
& +\underbrace{B\left[1-\sum_{I=1}^{N} \lambda_{l}(\mathbf{x})\right]^{2}}_{\text {Drives the } \lambda_{l} \text { to sum to } 1} .
\end{aligned}
$$

To understand this expression, consider a point $\mathbf{x}$ and no stress. The second term drives every variant $I$ to be represented by either $\lambda_{I}=0$ or $\lambda_{I}=1$. The third term drives the sum of $\lambda_{I}$ at a point to be 1 . Taken together, these terms provide a driving force for one and only one variant $K$ to have $\lambda_{K}=1$ and the other $\lambda_{J}=0, J \neq K$. Given this distribution of $\left\{\lambda_{I}\right\}$, the stress-free strain $\boldsymbol{\epsilon}_{0}=\sum_{I=1}^{N} \lambda_{I} \boldsymbol{\epsilon}_{0}^{I}$ will be just the stress-free strain $\boldsymbol{\epsilon}_{0}^{K}$ of the variant that is represented by $\lambda_{K}=1$, and the elastic energy is minimized when $\boldsymbol{\epsilon}=\boldsymbol{\epsilon}_{0}^{K}$.

There are two key innovations of the general approach. The first is the use of separate driving forces for each $\lambda_{I}$ to drive them to 0 or 1 in the second term. The separate driving forces inherently satisfy the symmetry requirements, and the procedure is the same for any crystal symmetry. This is much simpler than the polynomials that are typically used in phasefield modeling. ${ }^{7,15}$ While we use a specific choice for demonstration, any driving force that drives each $\lambda_{I}$ to 0 or 1 can be used. For accurate fitting of energy surfaces of specific materials, other choices can be made, e.g., $\lambda_{I}^{4}\left(1-\lambda_{I}\right)^{4}$ lowers 
the energy barriers. The second innovation is the use of a penalty to require the $\lambda_{I}$ to sum to 1 . Again, we use a specific choice but other expressions that fit the energy better are possible, as long as it drives the sum to 1 .

Our approach is closely related to and builds on the work of Shu and co-workers; ${ }^{8-10}$ they introduce the variables $\lambda_{I}$ and then transform them to a new set motivated by laminate microstructures. However, this transformation is not symmetric in the variants and hence the crystallographic symmetry is not satisfied. The energy that we have written above is obviously symmetric. An additional advantage of our approach (inherited from Refs. 8-10) is that there are no strain gradients that would require higher-order elements for a finite-element implementation. ${ }^{17}$

The microstructure is obtained by minimizing the energy in Eq. (1). This is done by evolving from the initial state following the variational derivative of $E$ with respect to $\lambda_{I}$ :

$$
\begin{aligned}
\frac{1}{\mu} \frac{\partial \lambda_{I}}{\partial t}=-\frac{\delta E}{\delta \lambda_{I}}= & A_{0} \nabla^{2} \lambda_{I}-2 A \lambda_{I}\left(1-\lambda_{I}\right)\left(1-2 \lambda_{I}\right) \\
& -2 B\left(\sum_{J=1}^{N} \lambda_{J}-1\right)+\boldsymbol{\epsilon}_{0}^{I}: \mathbf{C}:\left(\boldsymbol{\epsilon}-\boldsymbol{\epsilon}_{0}\right) .
\end{aligned}
$$

While evolving, at each step we set the variational derivative of $E$ with respect to $\boldsymbol{\epsilon}$ to $\mathbf{0}$

$$
\frac{\delta E}{\delta \boldsymbol{\epsilon}}=\nabla \cdot[\underbrace{\mathbf{C}:\left(\boldsymbol{\epsilon}-\boldsymbol{\epsilon}_{0}\right)}_{\text {stress }}]=\mathbf{0} .
$$

This ensures mechanical equilibrium throughout the evolution and at the final state.

We use standard phase-field numerical techniques to solve the resulting evolution and elasticity equations. The time-evolution uses a semi-explicit scheme, and the elasticity uses a fast Fourier technique with periodic boundary conditions. For details, we refer the reader to the Appendix of the work by Shu and Yen. ${ }^{8}$ Normalizing all length scales by the interface thickness, the computational domain is a square of size 100 and the mesh spacing is 0.1 . The normalized time; $\hat{t}=(2 \mu A) t$ and the time step is $\Delta \hat{t}=0.01$.

We apply our formulation to predict microstructure in shape-memory cubic-to-monoclinic nickel titanium (NiTi) with thin-film geometry. In NiTi with cubic to monoclinic phase transformation, there are 12 variants. The stress-free strains of the variants are as follows:

$$
\begin{aligned}
& \boldsymbol{\epsilon}_{0}^{1} \equiv\left(\begin{array}{ccc}
\epsilon_{\beta} & \epsilon & \epsilon \\
\epsilon & \epsilon_{\alpha} & \delta \\
\epsilon & \delta & \epsilon_{\alpha}
\end{array}\right) \boldsymbol{\epsilon}_{0}^{2} \equiv\left(\begin{array}{ccc}
\epsilon_{\beta} & -\epsilon & -\epsilon \\
-\epsilon & \epsilon_{\alpha} & \delta \\
-\epsilon & \delta & \epsilon_{\alpha}
\end{array}\right) \\
& \boldsymbol{\epsilon}_{0}^{3} \equiv\left(\begin{array}{ccc}
\epsilon_{\beta} & -\epsilon & \epsilon \\
-\epsilon & \epsilon_{\alpha} & -\delta \\
\epsilon & -\delta & \epsilon_{\alpha}
\end{array}\right) \boldsymbol{\epsilon}_{0}^{4} \equiv\left(\begin{array}{ccc}
\epsilon_{\beta} & \epsilon & -\epsilon \\
\epsilon & \epsilon_{\alpha} & -\delta \\
-\epsilon & -\delta & \epsilon_{\alpha}
\end{array}\right) \\
& \boldsymbol{\epsilon}_{0}^{5} \equiv\left(\begin{array}{ccc}
\epsilon_{\alpha} & \epsilon & \delta \\
\epsilon & \epsilon_{\beta} & \epsilon \\
\delta & \epsilon & \epsilon_{\alpha}
\end{array}\right) \boldsymbol{\epsilon}_{0}^{6} \equiv\left(\begin{array}{ccc}
\epsilon_{\alpha} & -\epsilon & \delta \\
-\epsilon & \epsilon_{\beta} & -\epsilon \\
\delta & -\epsilon & \epsilon_{\alpha}
\end{array}\right)
\end{aligned}
$$

$$
\begin{gathered}
\boldsymbol{\epsilon}_{0}^{7} \equiv\left(\begin{array}{ccc}
\epsilon_{\alpha} & -\epsilon & -\delta \\
-\epsilon & \epsilon_{\beta} & \epsilon \\
-\delta & \epsilon & \epsilon_{\alpha}
\end{array}\right) \boldsymbol{\epsilon}_{0}^{8} \equiv\left(\begin{array}{ccc}
\epsilon_{\alpha} & \epsilon & -\delta \\
\epsilon & \epsilon_{\beta} & -\epsilon \\
-\delta & -\epsilon & \epsilon_{\alpha}
\end{array}\right) \\
\boldsymbol{\epsilon}_{0}^{9} \equiv\left(\begin{array}{ccc}
\epsilon_{\alpha} & \delta & \epsilon \\
\delta & \epsilon_{\alpha} & \epsilon \\
\epsilon & \epsilon & \epsilon_{\beta}
\end{array}\right) \boldsymbol{\epsilon}_{0}^{10} \equiv\left(\begin{array}{ccc}
\epsilon_{\alpha} & \delta & -\epsilon \\
\delta & \epsilon_{\alpha} & -\epsilon \\
-\epsilon & -\epsilon & \epsilon_{\beta}
\end{array}\right) \\
\boldsymbol{\epsilon}_{0}^{11} \equiv\left(\begin{array}{ccc}
\epsilon_{\alpha} & -\delta & \epsilon \\
-\delta & \epsilon_{\alpha} & -\epsilon \\
\epsilon & -\epsilon & \epsilon_{\beta}
\end{array}\right) \epsilon_{0}^{12} \equiv\left(\begin{array}{ccc}
\epsilon_{\alpha} & -\delta & -\epsilon \\
-\delta & \epsilon_{\alpha} & \epsilon \\
-\epsilon & \epsilon & \epsilon_{\beta}
\end{array}\right),
\end{gathered}
$$

where $\epsilon_{\alpha}=0.0243, \epsilon_{\beta}=-0.0437, \delta=0.058, \epsilon=-0.0427,{ }^{1}$ and material constants $A_{0}=2 \times 10^{-4}, A=0.1270, B=0.1270$, $C_{11}=C_{22}=80 \mathrm{GPa}, C_{12}=20 \mathrm{GPa}, C_{66}=30 \mathrm{GPa}, C_{16}=C_{26}$ $=0 \mathrm{GPa}^{8}$

We examine a (111) deposited thin film and the texture is introduced by replacing the expressions in Eq. (5) by $\mathbf{R}_{(111)} \boldsymbol{\epsilon}_{0}^{I} \mathbf{R}_{(111)}^{T}$ where $\mathbf{R}_{(111)}$ is the proper rotation that maps to this texture. Further, in the thin-film limit, out-of-plane mechanical compatibility does not play a role and only the inplane strains are of importance. ${ }^{6}$ We orient the film in the 1-2 plane and hence only the components $\left(\epsilon_{0}^{I}\right)_{11},\left(\epsilon_{0}^{I}\right)_{12}$, $\left(\epsilon_{0}^{I}\right)_{21}$, and $\left(\epsilon_{0}^{I}\right)_{22}$ are used. Considering the simulation domain as a small portion of a thin-film specimen, we apply periodic boundary conditions with displacement boundary conditions. We predict various microstructures as shown in Fig. 1.

Interesting results from the calculations include that the same far field applied strain causes two possible crossing twin microstructures when the space-averaged volume fractions of all four participating variants is equal to 0.25 . In Fig. 1 (a), a far-field applied strain of $\left[\begin{array}{c}0.0330 \\ 0.0\end{array}-0.0013\right]$ leads to a crossing twin microstructure between the variants $8,4,5$, and 1 . The same far-field strain can also lead to a crossing twin microstructure with the same variants but different geometry, Fig. 1(b). Both microstructures are compatible and completely stress-free. This degeneracy in microstructure can provide shape-memory alloys with multiple low-energy possibilities to accommodate certain macroscopic deformations.

When the far-field strain is changed to $\left[\begin{array}{c}0.0330 \\ 0.0\end{array}-0.0048\right.$, the martensitic microstructure in Fig. 1(a) evolves to a new pattern as in Fig. 1(c). However, the microstructure in Fig. 1(b) is unable to accommodate this new applied strain in a stressfree manner. Instead, extremely large stresses are seen, and would likely lead to plasticity or completely new microstructure.

Figure 1(d) shows a stress-free microstructure under a

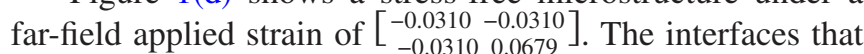
are formed are possible only in thin films and not in the bulk. $^{16}$

We also examine the microstructure in a (100) deposited thin film by using $\mathbf{R}_{(100)}$ in the expressions above and restricting attention to the 1-2 plane. In this setting, many of the variants have the same inplane stress-free strains, and only six distinct variants remain. Figure 2 shows a fourvariant crossing twin stress-free microstructure under a farfield applied strain of $\left[\begin{array}{cc}0.0045 & -0.0113 \\ -0.0113 & -0.0239\end{array}\right]$. This provides an interesting comparison between bulk microstructures and thinfilm microstructures. Variants 1, 2, 7, and 5 can form a 


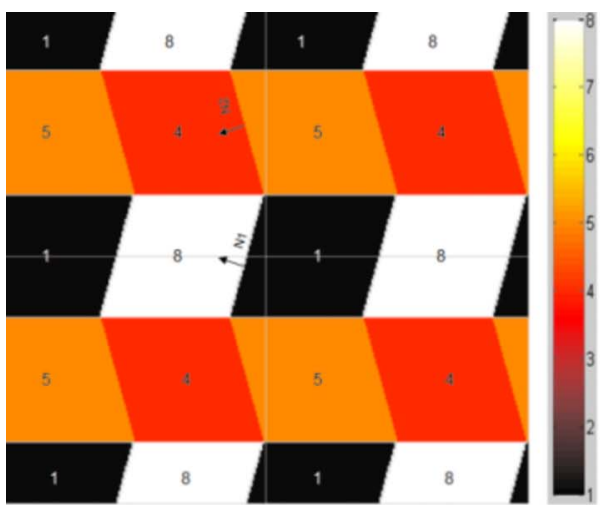

(a)

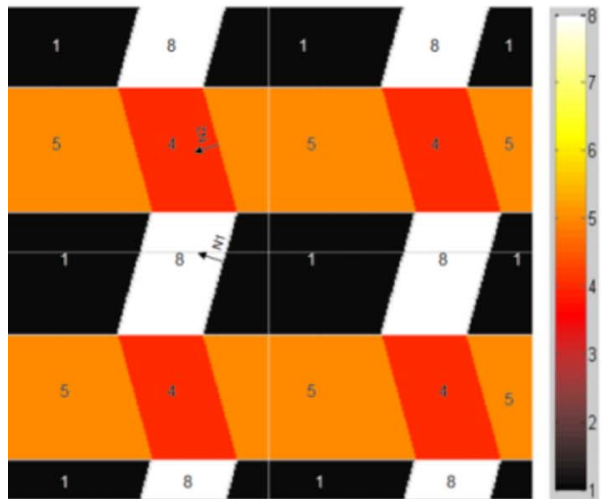

(c)

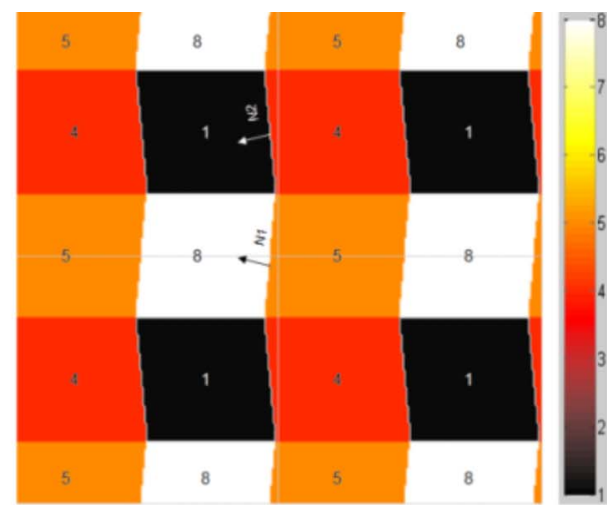

(b)

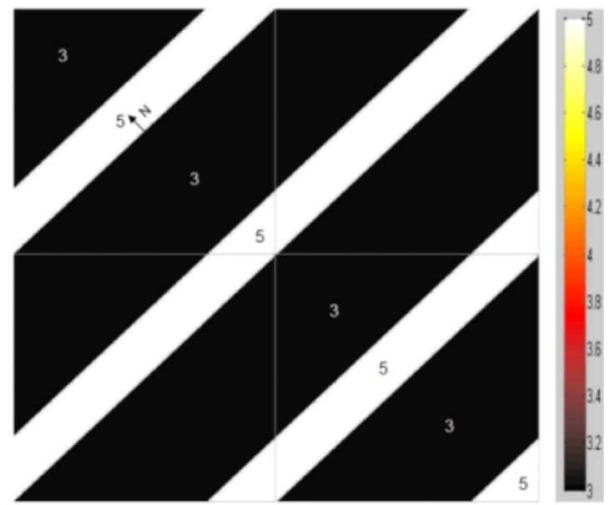

(d)

FIG. 1. (Color online) Microstructure in (111) deposited NiTi thin films. Plot shows the field $\Sigma_{I=1}^{N} I \times \lambda_{I}(\mathbf{x})$. (a) Crossing twin microstructure between variants $8,4,5$, and 1 . The interface normals are $(0.968, \pm 0.252)$ (Type II irrational twins). (b) Alternative crossing twin microstructure between variants $8,4,5$, and 1. The interface normals are $(0.996, \pm 0.088)$ (Type II irrational twins). (c) Crossing twin microstructure between variants $8,4,5$, and 1 with nonequal volume fractions. (d) Microstructure between variants 3 and 5 that is possible only in thin films and not in bulk specimens. The interface normal is $(1,1)$.

stress-free microstructure of this type in the bulk setting. However, in the (100) thin film, variant 6 and variant 7 have the same $\left(\epsilon_{0}^{I}\right)_{11},\left(\epsilon_{0}^{I}\right)_{12},\left(\epsilon_{0}^{I}\right)_{21}$, and $\left(\epsilon_{0}^{I}\right)_{22}$ components. Hence, in thin films this microstructure can also represent a crossing twin between variants $1,2,6$, and 5 . The crossing twin microstructure between variants $1,2,6$, and 5 is possible only in the thin film but would cause stresses in a bulk specimen.

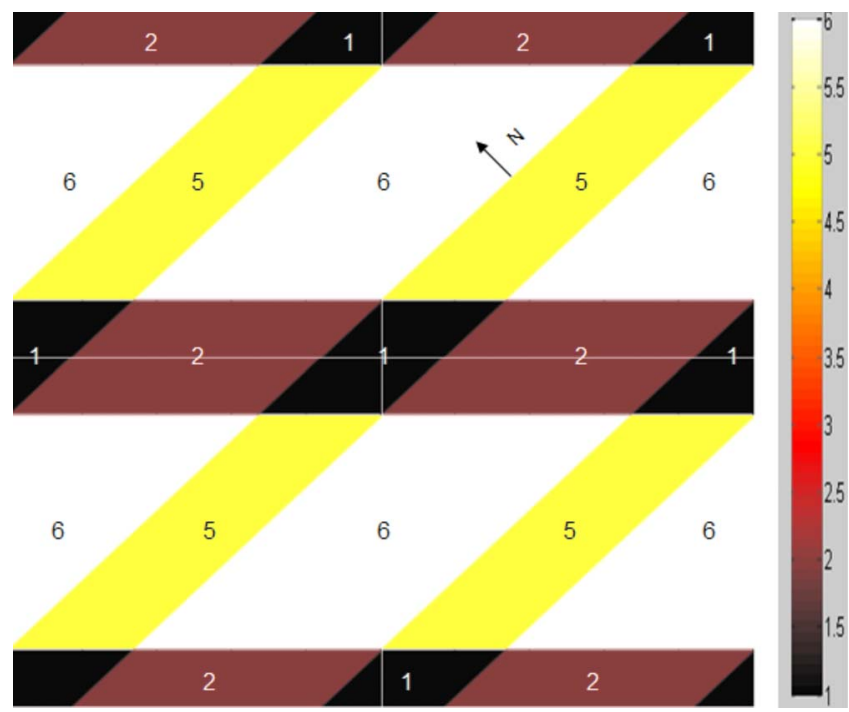

FIG. 2. (Color online) Stress-free microstructure in (100) thin film. The interface normals are $(1,1)$. In this film geometry, it can be interpreted as a microstructure involving variants $1,2,6$, and 5 which is only possible in thin film, or as a microstructure involving variants $1,2,7$, and 5 that is possible in both bulk and thin film. Plot shows the field $\sum_{I=1}^{N} I \times \lambda_{I}(\mathbf{x})$.
The method can be extended to ferroelectric and ferromagnetic materials, e.g., by adding the contribution (p $\left.-\mathbf{p}_{0}\right) \cdot \chi^{-1} \cdot\left(\mathbf{p}-\mathbf{p}_{0}\right)$ to $W_{\text {aniso, }}$, and using $\mathbf{p}_{0}(\mathbf{x})=\sum_{I=1}^{N} \lambda_{I}(\mathbf{x}) \mathbf{p}_{0}^{I}$ where $\mathbf{p}$ is the polarization and $\mathbf{p}_{0}^{I}$ is the spontaneous polarization of each variant. An important question for future research is to accurately fit the constants and functions to model the transformation barriers and other material information, e.g., (2.40) in Ref. 18.

We thank Yi-Chung Shu for useful discussions.

${ }^{1} \mathrm{~K}$. Bhattacharya, Microstructure of Martensite (Oxford University Press, Oxford, 2003).

${ }^{2}$ F. Jona and G. Shirane, Ferroelectric Crystals (Pergamon, Oxford, 1962).

${ }^{3} \mathrm{Y}$. Xu, Ferroelectric Materials and Their Applications (North-Holland, Amsterdam, 1991).

${ }^{4}$ K. Dayal and K. Bhattacharya, J. Appl. Phys. 102, 064102 (2007).

${ }^{5}$ L. Zhang, L. Q. Chen, and Q. Du, Acta Mater. 56, 3568 (2008).

${ }^{6}$ L. Li, J. Li, Y. Shu, and J. Yen, Appl. Phys. Lett. 93, 192506 (2008).

${ }^{7}$ L. Q. Chen, Annu. Rev. Mater. Res. 32, 113 (2002).

${ }^{8}$ Y. C. Shu and J. H. Yen, Acta Mater. 56, 3969 (2008).

${ }^{9}$ Y. C. Shu and J. H. Yen, Appl. Phys. Lett. 91, 021908 (2007).

${ }^{10}$ Y. C. Shu, J. H. Yen, H. Z. Chen, J. Y. Li, and L. J.Li, Appl. Phys. Lett. 92, 052909 (2008).

${ }^{11}$ R. Ahluwalia, T. Lookman, A. Saxena, and W. Cao, Phys. Rev. B 72 014112 (2005).

${ }^{12}$ K. Dayal and K. Bhattacharya, Acta Mater. 55, 1907 (2007).

${ }^{13}$ M. Y. El-Naggar, K. Dayal, D. G. Goodwin, and K. Bhattacharya, J. Appl. Phys. 100, 114115 (2006).

${ }^{14}$ K. Hormann and J. Zimmer, J. Mech. Phys. Solids 55, 1385 (2007).

${ }^{15}$ Y. Su and C. Landis, J. Mech. Phys. Solids 55, 280 (2007).

${ }^{16}$ K. Bhattacharya and R. James, J. Mech. Phys. Solids 47, 531 (1999).

${ }^{17}$ R. Abeyaratne and J. Knowles, SIAM J. Appl. Math. 51, 1205 (1991).

${ }^{18}$ C. Landis, J. Mech. Phys. Solids 56, 3059 (2008). 